\title{
SOBOLEV SPACE PROJECTIONS IN STRICTLY PSEUDOCONVEX DOMAINS ${ }^{1}$
}

BY

HAROLD P. BOAS

\begin{abstract}
The orthogonal projection from a Sobolev space $W^{s}(\Omega)$ onto the subspace of holomorphic functions is studied. This analogue of the Bergman projection is shown to satisfy regularity estimates in higher Sobolev norms when $\Omega$ is a smooth bounded strictly pseudoconvex domain in $\mathbf{C}^{n}$.
\end{abstract}

The Bergman projection $P_{0}: L^{2}(\Omega) \rightarrow L^{2}(\Omega) \cap$ \{holomorphic functions , where $\Omega \subset \mathbf{C}^{n}$ is a smooth bounded domain, has proved to be a key element in the study of boundary behavior of holomorphic mappings (see $[4,7,13]$ and their references). In the important special case in which $\Omega$ is strictly pseudoconvex, a great deal is known about the projection $P_{0}$ and the Bergman kernel function $K_{0}(w, z)$ which represents it (see e.g. $[14,16,19])$. In particular the following two regularity theorems are well known consequences of Kohn's theory of the $\bar{\partial}$-Neumann problem [15, 17].

TheOREM A [17]. Let $\Omega \subset \mathbf{C}^{n}$ be a smooth bounded strictly pseudoconvex domain. Then the Bergman projection $P_{0}$ admits both global and local regularity estimates in Sobolev norms:

$$
\left\|P_{0} u\right\|_{r} \leqslant C_{r}\|u\|_{r}, \quad r \geqslant 0,
$$

and more generally, if $\zeta_{1}, \zeta_{2} \in C_{0}^{\infty}\left(\mathbf{C}^{n}\right)$ are real-valued cut-off functions with $\zeta_{2}=1$ in a neighborhood of the support of $\zeta_{1}$, then

$$
\left\|\zeta_{1} P_{0} u\right\|_{r} \leqslant C_{r}\left(\left\|\zeta_{2} u\right\|_{r}+\|u\|_{0}\right), \quad r \geqslant 0
$$

THEOREM B [16]. Let $\Omega \subset \mathbf{C}^{n}$ be a smooth bounded strictly pseudoconvex domain. Then the Bergman kernel function $K_{0}(w, z)$ is smooth up to the boundary off the boundary diagonal, that is,

$$
K_{0}(w, z) \in C^{\infty}(\bar{\Omega} \times \bar{\Omega} \backslash\{z=w \in b \Omega\}) .
$$

The objects studied in this paper are the analogous projection $P_{s}: W^{s}(\Omega) \rightarrow$ $W^{s}(\Omega) \cap\{$ holomorphic functions , where $\Omega$ is a smooth bounded strictly pseudoconvex domain and $W^{s}(\Omega)$ is the Sobolev space of functions with $s$ square-integrable

Received by the editors October 24, 1983 and, in revised form, May 29, 1984.

1980 Mathematics Subject Classification. Primary 32A25, 32H10.

Kev words and phrases. Bergman kernel function, $\bar{\partial}$-Neumann problem, pseudoconvex domains.

${ }^{1}$ This material is partially based on work supported by the National Science Foundation under Grant No. MCS-8201063. 
derivatives, and the kernel function $K_{s}(w, z)$ that represents $P_{s}$. The main result is that the analogues of Theorems $\mathrm{A}$ and $\mathrm{B}$ hold.

TheOrem $\mathrm{A}_{s}$. Let $\Omega \subset \mathbf{C}^{n}$ be a smooth bounded strictly pseudoconvex domain. Then the projection $P_{s}$ admits both global and local regularity estimates in Sobolev norms:

$$
\left\|P_{s} u\right\|_{r} \leqslant C_{r . s}\|u\|_{r}, \quad r \geqslant s,
$$

and more generally, if $\zeta_{1}, \zeta_{2} \in C_{0}^{x}\left(\mathbf{C}^{n}\right)$ are real-valued cut-off functions with $\zeta_{2}=1$ in a neighborhood of the support of $\zeta_{1}$, then

$$
\left\|\zeta_{1} P_{s} u\right\|_{r} \leqslant C_{r, s}\left(\left\|\zeta_{2} u\right\|_{r}+\|u\|_{s}\right), \quad r \geqslant s
$$

THEOREM $\mathrm{B}_{s}$. Let $\Omega \subset \mathbf{C}^{n}$ be a smooth bounded strictly pseudoconvex domain. Then the kernel function $K_{s}(w, z)$ is smooth up to the boundary off the boundary diagonal, that is

$$
K_{s}(w, z) \in C^{\infty}(\bar{\Omega} \times \bar{\Omega} \backslash\{z=w \in b \Omega\}) .
$$

The key to understanding the projection $P_{s}$ is to prove regularity estimates for the operators $U_{s}$ and $T_{s}$ given by integration against a kernel in the "wrong" space:

$$
U_{s} u(w)=\left\langle u(\cdot), K_{s}(\cdot, w)\right\rangle_{0}, \quad T_{s} u(w)=\left\langle u(\cdot), K_{0}(\cdot, w)\right\rangle_{s} .
$$

From these operators, first mentioned by Bell [5], one recaptures $P_{s}$ via the formula $P_{s}=U_{s} T_{s}$.

The key estimates for $U_{s}$ and $T_{s}$ are proved in $\$ 5$ using results from the $\bar{\partial}$-Neumann theory. The proofs of Theorems $\mathrm{A}_{s}$ and $\mathrm{B}_{s}$, based on these estimates, are given in $\$ \S 2$ and 3: the global part in $\$ 2$ and the more delicate local part in $\S 3$.

I thank N. Kerzman, who suggested study of the $K_{s}$ kernel as a thesis problem. My MIT doctoral dissertation, written under his direction, was a first step toward the results presented here. The papers of S. Bell, especially [5], have also provided inspiration in this work.

1. Preliminaries. Throughout, $\Omega$ is a smooth bounded strictly pseudoconvex domain in $\mathbf{C}^{n}$. This means that there is a bounded $C^{\infty}$ real-valued defining function $\rho: \mathbf{C}^{n} \rightarrow \mathbf{R}$ such that $\Omega=\left\{z \in \mathbf{C}^{n}: \rho(z)<0\right\}$, the boundary $b \Omega=\left\{z \in \mathbf{C}^{n}: \rho(z)\right.$ $=0\}$, the gradient of $\rho$ does not vanish on $b \Omega$, and the complex Hessian of $\rho$ is strictly positive definite on the complex part of the tangent space. It will be assumed that $\rho$ is normalized so that the gradient of $\rho$ has length one on the boundary. In that case the globally defined vector field $\partial / \partial \rho$ given in the underlying real coordinates by

$$
\frac{\partial}{\partial \rho}=\sum_{j=1}^{2 n} \frac{\partial \rho}{\partial x_{j}} \frac{\partial}{\partial x_{j}}
$$

agrees on the boundary with the unit outer normal.

The Hilbert space $L^{2}(\Omega)$ of square-integrable functions on $\Omega$ carries the usual norm \|\|$_{0}$ induced by the inner product $\langle u, v\rangle_{0}=\int_{\Omega} u \bar{v}$. When $s$ is a positive integer the inner product

$$
\langle u, v\rangle_{s}=\sum_{|\alpha| \leqslant s}\left\langle D^{\alpha} u, D^{\alpha} v\right\rangle_{0}
$$


induces the norm \|\|$_{s}$ on the Sobolev space $W^{s}(\Omega)$. (Here $\alpha=\left(\alpha_{1}, \ldots, \alpha_{2 n}\right)$ is a multi-index and $D_{j}=\partial / \partial x_{j}$.) When $s$ is a positive real number that is not an integer, $W^{s}(\Omega)$ can be defined by an interpolation procedure (see e.g. [18]). The closure in $W^{s}(\Omega)$ of the space $C_{0}^{\infty}(\Omega)$ of smooth functions with compact support in $\Omega$ is denoted $W_{0}^{s}(\Omega)$. The intersection of all the spaces $W^{s}(\Omega)$, taken with the usual inverse limit topology, is the space $C^{\infty}(\bar{\Omega})$ of functions smooth up to the boundary; it is a dense subspace of each $W^{s}(\Omega)$.

The space $W^{-s}(\Omega)$, defined for each positive real number $s$, is the dual space of $W_{0}^{s}(\Omega)$. It is realized as a space of distributions containing $L^{2}(\Omega)$ as a dense subspace. If $u \in L^{2}(\Omega)$, then

$$
\|u\|_{-s}=\sup \left\{\left|\langle u, \varphi\rangle_{0}\right|: \varphi \in C_{0}^{\infty}(\Omega),\|\varphi\|_{s}=1\right\} .
$$

The completion of $L^{2}(\Omega)$ in the stronger norm

$$
\|u\|_{-s}^{*}=\sup \left\{\left|\langle u, v\rangle_{0}\right|: v \in C^{\infty}(\bar{\Omega}),\|v\|_{s}=1\right\}
$$

is the dual space $\left(W^{s}(\Omega)\right)^{*}$ of $W^{s}(\Omega)$; it is not in general identified with a space of distributions. Always $\|u\|_{-s} \leqslant\|u\|_{-s}^{*}$, and if $u$ is holomorphic it turns out that the two norms are equivalent (see Lemma 4.4).

The elements of $W^{s}(\Omega)$ represented by holomorphic functions comprise a closed subspace $H^{s}(\Omega)$. (N.B. This notation conflicts with common usage, in which $H^{s}$ denotes the usual Sobolev space. It is convenient here to reserve the letter $H$ to indicate a space of holomorphic functions.) The intersection of all the spaces $H^{s}(\Omega)$ with the topology inherited from $C^{\infty}(\bar{\Omega})$ is denoted $H^{\infty}(\bar{\Omega})$. The union of the spaces $H^{-s}(\Omega)$ with the usual inductive limit topology is denoted $H^{-\infty}(\Omega)$.

The objects of interest in this paper are, for each positive integer $s$, the orthogonal projection $P_{s}: W^{s}(\Omega) \rightarrow H^{s}(\Omega)$ and the kernel function $K_{s}(w, z)$ that represents it. When $u \in W^{s}(\Omega)$

$$
P_{s} u(z)=\left\langle u(\cdot), K_{s}(\cdot, z)\right\rangle_{s}
$$

and in particular for every holomorphic function $h$ in $H^{s}(\Omega)$ the reproducing property $h(z)=\left\langle h(\cdot), K_{s}(\cdot, z)\right\rangle_{s}$ holds. For some elementary properties of $K_{s}$ see [9], and for the general theory of reproducing kernels see [2].

The central idea is to relate the projection $P_{s}$ to the usual Bergman projection $P_{0}$. A principal tool is the following integration by parts lemma, which holds in an arbitrary smooth bounded domain $\Omega$ (not necessarily pseudoconvex).

LEMMA 1.1. For each positive integer $s$ there is a linear differential operator $L^{2 s}$ of order $2 s$ with coefficients in $C^{\infty}(\bar{\Omega})$ such that for every holomorphic function $h$ in $H^{s}(\Omega)$ and every function $u$ in $W^{2 s}(\Omega)$

$$
\langle h, u\rangle_{s}=\left\langle h, L^{2 s} u\right\rangle_{0} .
$$

If $u$ is also holomorphic, then the top order term of $L^{2 s} u$ reduces to $2^{s}(\partial / \partial \rho)^{2 s} u$.

Proof. Suppose at first that $h \in H^{\infty}(\bar{\Omega})$ and $u \in C^{\infty}(\bar{\Omega})$. Derivatives of $h$ that are tangential near the boundary can be integrated by parts with no boundary terms appearing, and by the Cauchy-Riemann equations normal derivatives of the holomorphic function $h$ can be rewritten as tangential derivatives. Hence all derivatives 
on $h$ can be moved to the other side of the inner product to make formula (1.1) appear.

To identify the top order term of $L^{2 s}$ observe that by Green's identity

$$
\begin{aligned}
\langle h, u\rangle_{1} & =\int_{\Omega} h \bar{u}+\int_{\Omega} \sum_{j=1}^{2 n}\left(D_{j} h\right)\left(D_{j} \bar{u}\right) \\
& =\int_{\Omega} h \bar{u}+\int_{b \Omega} \frac{\partial h}{\partial \rho} \bar{u},
\end{aligned}
$$

where Lebesgue measure is understood both on the boundary and on the interior. There is a real vector field $\partial / \partial \sigma$ on $\Omega$, tangential at the boundary, with coefficients in $C^{\infty}(\bar{\Omega})$, such that $(\partial / \partial \rho)+i(\partial / \partial \sigma)$ is a vector field of type $(1,0)$. Then $\partial h / \partial \rho=i \partial h / \partial \sigma$, so integration by parts and another application of Green's identity give

$$
\langle h, u\rangle_{1}=\left\langle h,\left(1+i(\Delta \rho) \frac{\partial}{\partial \sigma}-\frac{\partial^{2}}{\partial \sigma^{2}}+i \frac{\partial}{\partial \rho} \frac{\partial}{\partial \sigma}\right) u\right\rangle_{0},
$$

where $\Delta$ is the usual Laplace operator. It follows by induction that the leading term of $L^{2 s}$ is

$$
\left(-\frac{\partial^{2}}{\partial \sigma^{2}}+i \frac{\partial}{\partial \rho} \frac{\partial}{\partial \sigma}\right)^{s}
$$

which, when applied to a holomorphic function, reduces to $2^{s}(\partial / \partial \rho)^{2 s}$ plus lower order terms.

Since $C^{\infty}(\bar{\Omega})$ is dense in $W^{2 s}(\Omega)$, equation (1.1) continues to hold when $u$ is only in $W^{2 s}(\Omega)$. Passage to the limit over the interior approximating domains $\Omega_{\delta}=\{z \in$ $\Omega: \rho(z)<-\delta\}$ shows that (1.1) persists also for $h$ only in $H^{s}(\Omega)$.

2. Global regularity and duality. When $u \in L^{2}(\Omega)$, a holomorphic function $U_{s} u$ is defined by

$$
U_{s} u(w)=\left\langle u(\cdot), K_{s}(\cdot, w)\right\rangle_{0} .
$$

By Theorem B (Kerzman [16]) the Bergman kernel function $K_{0}(\cdot, w) \in C^{\infty}(\bar{\Omega})$ in every smooth bounded strictly pseudoconvex domain $\Omega$, so when $u \in W^{s}(\Omega)$ a holomorphic function $T_{s} u$ is defined by

$$
T_{s} u(w)=\left\langle u(\cdot), K_{0}(\cdot, w)\right\rangle_{s} .
$$

The operators $U_{s}$ and $T_{s}$, denoted $L^{s}$ and $\Lambda^{s}$ by Bell [5], are important because composing them recovers the projections $P_{0}$ and $P_{s}$.

LEMMA 2.1. (a) The operator $T_{s}$ maps $C^{\infty}(\bar{\Omega})$ continuously into itself and $U_{s} T_{s}=P_{s}$ on $C^{\infty}(\bar{\Omega})$.

(b) The operator $U_{s}$ maps $L^{2}(\Omega)$ continuously into $H^{s}(\Omega)$ and $T_{s} U_{s}=P_{0}$ on $L^{2}(\Omega)$.

Proof. By Lemma 1.1 the equation $T_{s} u=P_{0} L^{2 s} u$ holds for $u$ in $C^{\infty}(\bar{\Omega})$. Since the Bergman projection $P_{0}$ for a smooth bounded strictly pseudoconvex domain maps $C^{\infty}(\bar{\Omega})$ continuously into itself, it follows that $T_{s}$ has the same property. In particular 
$U_{s} T_{s} u$ is defined for $u$ in $C^{\infty}(\bar{\Omega})$. Moreover

$$
\begin{aligned}
U_{s} T_{s} u(w) & =U_{s} P_{0} L^{2 s} u(w)=\left\langle P_{0} L^{2 s} u(\cdot), K_{s}(\cdot, w)\right\rangle_{0} \\
& =\left\langle L^{2 s} u(\cdot), K_{s}(\cdot, w)\right\rangle_{0}=\left\langle u(\cdot), K_{s}(\cdot, w)\right\rangle_{s}=P_{s} u(w) .
\end{aligned}
$$

This proves part (a).

Suppose $u \in C_{0}^{\infty}(\Omega)$. Since the norm of $K_{s}(z, \cdot)$ in $W^{s}(\Omega)$ is bounded by a constant depending on the distance from $z$ to the boundary $b \Omega$, the holomorphic function $U_{s} u$ lies in $H^{s}(\Omega)$. Moreover if $h \in H^{s}(\Omega)$, Fubini's theorem implies that

$$
\left\langle U_{s} u, h\right\rangle_{s}=\langle u, h\rangle_{0} \text {. }
$$

Therefore $\left\|U_{s} u\right\|_{s} \leqslant\|u\|_{0}$ for $u$ in $C_{0}^{\infty}(\Omega)$. Since this space is dense in $L^{2}(\Omega)$ it follows that $U_{s}$ is bounded from $L^{2}(\Omega)$ into $H^{s}(\Omega)$ and that equation (2.1) persists for $u$ in $L^{2}(\Omega)$. Substituting $K_{0}(z, \cdot)$ for $h(z)$ in (2.1) shows that $P_{0}$ equals $T_{s} U_{s}$ on $L^{2}(\Omega)$, which proves part (b).

In view of Lemma 2.1 estimates for $P_{s}$ will follow directly from estimates for $U_{s}$ and $T_{s}$. The following global estimates hold for all $h$ in $H^{\infty}(\bar{\Omega})$ and every real number $r$ :

$$
\begin{gathered}
C^{-1}\|h\|_{r+2 s} \leqslant\left\|T_{s} h\right\|_{r} \leqslant C\|h\|_{r+2 s}, \\
C^{-1}\|h\|_{r} \leqslant\left\|U_{s} h\right\|_{r+2 s} \leqslant C\|h\|_{r}
\end{gathered}
$$

with $C$ independent of $h$. Thus $T_{s}$ loses $2 s$ derivatives and $U_{s}$ gains $2 s$ derivatives. These estimates will be proved at the end of $\$ 5$ as a corollary of the key local estimates.

Proof of the global Part of Theorem $\mathrm{A}_{s}$. If $u \in C^{\infty}(\bar{\Omega})$, then $P_{s} u=U_{s} T_{s} u=$ $U_{s} P_{0} L^{2 s} u$ by Lemmas 1.1 and 2.1. It is well known that the Bergman projection is bounded on $W^{r}(\Omega)$ for every nonnegative $r$. (See e.g. [19] and further discussion of $P_{0}$ in $\S 4$.) Therefore by (2.3)

$$
\left\|P_{s} u\right\|_{r} \leqslant C\left\|P_{0} L^{2 s} u\right\|_{r-2 s} \leqslant C\left\|L^{2 s} u\right\|_{r-2 s} \leqslant C\|u\|_{r}
$$

when $r \geqslant 2 s$. Density of $C^{\infty}(\bar{\Omega})$ in $W^{r}(\Omega)$ implies that $P_{s}$ is bounded on $W^{r}(\Omega)$ when $r \geqslant 2 s$. Since by definition $P_{s}$ is bounded on $W^{s}(\Omega)$, it follows by interpolation that $P_{s}$ is bounded on $W^{r}(\Omega)$ when $r \geqslant s$. This proves the first part of Theorem $\mathrm{A}_{s}$, granted estimate (2.3).

The estimates (2.2) and (2.3) imply in particular that $T_{s}$ and $U_{s}$ are continuous operators from $H^{\infty}(\bar{\Omega})$ into itself. In view of Lemma 2.1 the operators $T_{s}$ and $U_{s}$ are in fact mutually inverse isomorphisms of $H^{\infty}(\bar{\Omega})$ onto itself.

LEMMA 2.2 The space $H^{\infty}(\bar{\Omega})$ is dense in $H^{r}(\Omega)$ for every real number $r$.

This density lemma is true in arbitrary smooth bounded pseudoconvex domains (not necessarily strictly pseudoconvex): see [6] for a proof. Together with the a priori estimates (2.2) and (2.3) the lemma implies that $T_{s}$ and $U_{s}$ extend to mutually inverse isomorphisms of $H^{-\infty}(\Omega)$ onto itself; moreover the extensions give mutually inverse isomorphisms

$$
T_{s}: H^{r+s}(\Omega) \rightarrow H^{r-s}(\Omega), \quad U_{s}: H^{r-s}(\Omega) \rightarrow H^{r+s}(\Omega)
$$


for every real number $r$. This generalizes a result of Bell [5], who established the isomorphism when $r=0$.

Bell also showed that the $H^{0}$ pairing $\langle, \quad\rangle_{0}: H^{\infty}(\bar{\Omega}) \times H^{\infty}(\bar{\Omega}) \rightarrow \mathrm{C}$ extends uniquely to a continuous pairing of $H^{-\infty}(\Omega) \times H^{\infty}(\bar{\Omega})$ exhibiting the latter spaces as mutually dual. The same is true for the $H^{s}$ pairing $\langle,\rangle_{s}$ in view of the equation $\langle g, h\rangle_{s}=\left\langle T_{s} g, h\right\rangle_{0}$, true for $g$ and $h$ in $H^{\infty}(\bar{\Omega})$.

3. Proof of the main theorems. In view of the relation $U_{s} T_{s}=P_{s}$ the local estimates for $P_{s}$ will follow from local estimates for $U_{s}$ and $T_{s}$.

THEOREM 3.1 (The key local estimates). Let $\zeta_{1}$ and $\zeta_{2}$ be real-valued cut-off functions in $C_{0}^{\infty}\left(\mathbf{C}^{n}\right)$ such that $\zeta_{2}=1$ in a neighborhood of the support of $\zeta_{1}$.

(a) For every real number $r$ greater than or equal to s there is a constant $C$ such that

$$
\left\|\zeta_{1} T_{s} u\right\|_{r-2 s} \leqslant C\left(\left\|\zeta_{2} u\right\|_{r}+\|u\|_{s}\right)
$$

for all $u$ in $C^{\infty}(\bar{\Omega})$. For holomorphic functions a stronger estimate holds: for every real number $r$ (unrestricted) and every positive integer $M$ there is a constant $C$ such that

$$
\left\|\zeta_{1} T_{s} h\right\|_{r-2 s} \leqslant C\left(\left\|\zeta_{2} h\right\|_{r}+\|h\|_{-M}\right)
$$

for all $h$ in $H^{\infty}(\bar{\Omega})$.

(b) For every real number $r$ and every positive integer $M$ there is a constant $C$ such that

$$
\left\|\zeta_{1} h\right\|_{r} \leqslant C\left(\left\|\zeta_{2} T_{s} h\right\|_{r-2 s}+\|h\|_{-M}\right)
$$

for all $h$ in $H^{s}(\Omega)$, and

$$
\left\|\zeta_{1} U_{s} h\right\|_{r} \leqslant C\left(\left\|\zeta_{2} h\right\|_{r-2 s}+\|h\|_{-M}\right)
$$

for all $h$ in $H^{0}(\Omega)$.

REMARK. It is part of the theorem that finiteness of the right-hand side in (b) implies finiteness of the left-hand side. The upper bounds for $T_{s}$ follow easily from standard estimates for the Bergman projection. The lower bound for $T_{s}$ and corresponding upper bound for $U_{s}$ require some work. The proof is postponed until $\$ 5$.

THEOREM 3.2. Let $\zeta_{1}$ and $\zeta_{2}$ be real-valued cut-off functions in $C_{0}^{\infty}\left(\mathbf{C}^{n}\right)$ such that $\zeta_{2}=1$ in a neighborhood of the support of $\zeta_{1}$. If $u \in W^{s}(\Omega)$ and $\zeta_{2} u \in W^{r}(\Omega)$ for some real number $r$ greater than or equal to $s$, then $\zeta_{1} P_{s} u \in W^{r}(\Omega)$ and

$$
\left\|\zeta_{1} P_{s} u\right\|_{r} \leqslant C\left(\left\|\zeta_{2} u\right\|_{r}+\|u\|_{s}\right)
$$

with $C$ independent of $u$.

REMARK. This is a restatement of part (ii) of Theorem $\mathrm{A}_{s}$. Note that the statement reduces to part (i) if $\zeta_{1}$ is chosen to be identically 1 on $\Omega$.

Proof. Let $\eta$ be a smooth real-valued cut-off function such that $\eta=1$ in a neighborhood of the support of $\zeta_{1}$ and $\zeta_{2}=1$ in a neighborhood of the support of $\eta$. Suppose at first that $u \in C^{\infty}(\bar{\Omega})$. The relation $U_{s} T_{s}=P_{s}$ of Lemma 2.1 together with the upper bound (3.4) for $U_{s}$ implies

$$
\left\|\zeta_{1} P_{s} u\right\|_{r} \leqslant C\left(\left\|\eta T_{s} u\right\|_{r-2 s}+\left\|T_{s} u\right\|_{-s}\right) .
$$


Now estimate the right-hand side by applying inequality (3.1) twice: with $\zeta_{1}$ replaced by $\eta$ it gives

$$
\left\|\eta T_{s} u\right\|_{r-2 s} \leqslant C\left(\left\|\zeta_{2} u\right\|_{r}+\|u\|_{s}\right)
$$

and with the cut-off functions set equal to 1 on $\Omega$ it gives $\left\|T_{s} u\right\|_{-s} \leqslant C\|u\|_{s}$. Combining the last three equations shows that (3.5) holds as an a priori estimate for $u$ in $C^{\infty}(\bar{\Omega})$.

Now suppose only that $u \in W^{s}(\Omega)$ and $\zeta_{2} u \in W^{r}(\Omega)$. There is a sequence of functions $u_{1}, u_{2}, \ldots$ in $C^{\infty}(\bar{\Omega})$ such that $u_{j} \rightarrow u$ in $W^{s}(\Omega)$ and $\eta u_{j} \rightarrow \eta u$ in $W^{r}(\Omega)$ as $j \rightarrow \infty$. (Such functions can be constructed by means of a partition of unity and the standard bounded extension operator that extends a $W^{r}(\Omega)$ function supported in a boundary chart to a $W_{0}^{r}\left(\mathbf{C}^{n}\right)$ function.) The a priori version of (3.5) implies that as $j \rightarrow \infty$ the $\zeta_{1} P_{s} u_{j}$ converge in $W^{r}(\Omega)$ to some function $v$ such that

$$
\|v\|_{r} \leqslant C\left(\left\|\zeta_{2} u\right\|_{r}+\|u\|_{s}\right) .
$$

Since $P_{s}$ is by definition continuous in $W^{s}(\Omega)$, the $\zeta_{1} P_{s} u_{j}$ converge to $\zeta_{1} P_{s} u$ in $W^{s}(\Omega)$. By uniqueness of limits $v=\zeta_{1} P_{s} u$, and so $\zeta_{1} P_{s} u \in W^{r}(\Omega)$ and satisfies (3.5).

This completes the proof of Theorem $\mathrm{A}_{s}$ of the introduction. For compactly supported functions (which of course are not dense in $W^{r}(\Omega)$ when $r>1 / 2$ ) the index of the global term in (3.5) can be made arbitrary. This improvement is required to prove Theorem $\mathrm{B}_{s}$.

LeMma 3.3. Under the hypotheses of Theorem 3.2, if $\varphi \in C_{0}^{\infty}(\Omega)$ then for every positive integer $M$

$$
\left\|\zeta_{1} P_{s} \varphi\right\|_{r} \leqslant C\left(\left\|\zeta_{2} \varphi\right\|_{r}+\|\varphi\|_{-M}^{*}\right)
$$

with $C$ independent of $\varphi$.

REMARK. A stronger estimate holds: the index of the first term on the right-hand side can be reduced by $2 s$. However, this gain of derivatives is irrelevant in the application.

Proof. Since $\varphi$ has compact support it is possible to integrate by parts to obtain

$$
P_{s} \varphi(w)=\left\langle\varphi(\cdot), K_{s}(\cdot, w)\right\rangle_{s}=\left\langle\varphi(\cdot), K_{s}(\cdot, w)\right\rangle_{0}=U_{s} P_{0} \varphi(w) .
$$

If $\eta$ is an intermediate cut-off function as in the previous proof then the upper bound (3.4) for $U_{s}$ implies

$$
\left\|\zeta_{1} P_{s} \varphi\right\|_{r} \leqslant C\left(\left\|\eta P_{0} \varphi\right\|_{r}+\left\|P_{0} \varphi\right\|_{-M}\right) \leqslant C\left(\left\|\zeta_{2} \varphi\right\|_{r}+\|\varphi\|_{-M}^{*}\right) .
$$

The last step follows from standard estimates for the Bergman projection (see Lemma 4.1).

COROllary 3.4 (Theorem $\mathrm{B}_{s}$ ). For each positive integer $s$ the kernel function $K_{s}(w, z)$ is smooth up to the boundary off the boundary diagonal, that is

$$
K_{s}(w, z) \in C^{\infty}(\bar{\Omega} \times \bar{\Omega} \backslash\{(w, z) \in b \Omega \times b \Omega: w=z\}) .
$$


Proof. The case of the usual Bergman kernel function $(s=0)$ was established by Kerzman [16]. Because of Lemma 3.3 essentially the same argument works in the new setting. The details are as follows.

Of course $K_{s}(w, z) \in C^{\infty}(\Omega \times \Omega)$ since it is holomorphic in $w$ and conjugate holomorphic in $z$. What has to be checked is the behavior when $w$ approaches the boundary and $z$ stays away from $w$; possibly $z$ also approaches the boundary. By Sobolev's lemma it is enough to show that if $W$ is a small open set intersecting $b \Omega$ and $Z$ is an open set whose closure is disjoint from the closure of $W$, then

$$
\sup _{w \in W \cap \Omega}\left\|D_{w}^{\alpha} K_{s}(w, z)\right\|_{w^{r}(Z \cap \Omega)}<\infty
$$

for every multi-index $\alpha$ and every $r$ greater than $s$.

Let $\varphi$ be a smooth, nonnegative, radially symmetric function supported in the unit ball of $\mathbf{C}^{n}$ such that the integral of $\varphi$ over $\mathbf{C}^{n}$ equals 1. Define

$$
\varphi_{w}(t)=\operatorname{dist}(w, b \Omega)^{-2 n} \varphi((t-w) / \operatorname{dist}(w, b \Omega)) .
$$

For each $w$ in $\Omega$ the function $\varphi_{w}$ is smooth with compact support in $\Omega$, and the integral of $\varphi_{w}$ over $\mathbf{C}^{n}$ equals 1 . Integration by parts and the mean-value property of holomorphic functions imply

$$
(-1)^{|\alpha|} D_{w}^{\alpha} K_{s}(w, z)=\left\langle K_{s}(\cdot, z), D^{\alpha} \varphi_{w}(\cdot)\right\rangle_{0}=\overline{P_{s} D^{\alpha} \varphi_{w}(z)} .
$$

Let $\zeta$ be a smooth real-valued cut-off function that is identically zero in a neighborhood of the closure of $W$ and identically one in a neighborhood of the closure of $Z$. It is no loss of generality to assume that for every $w$ in $W$ the supports of $\varphi_{w}$ and $\zeta$ are disjoint. When $r \geqslant s$ and $w \in W$ it follows from the above formula and Lemma 3.3 that

$$
\left\|D_{w}^{\alpha} K_{s}(w, z)\right\|_{W^{r}(Z \cap \Omega)} \leqslant C\left(\left\|\zeta D^{\alpha} \varphi_{w}\right\|_{r}+\left\|D^{\alpha} \varphi_{w}\right\|_{-M}^{*}\right) \leqslant C\left\|D^{\alpha} \varphi_{w}\right\|_{-M}^{*}
$$

with $C$ independent of $w$. If $u \in C^{\infty}(\bar{\Omega})$, then

$$
\left|\left\langle D^{\alpha} \varphi_{w}, u\right\rangle_{0}\right|=\left|\left\langle\varphi_{w}, D^{\alpha} u\right\rangle_{0}\right| \leqslant\left(\int_{\Omega} \varphi_{w}\right) \sup _{\Omega}\left|D^{\alpha} u\right| \leqslant C\|u\|_{M}
$$

as soon as $M>n+|\alpha|$. Hence $\left\|D^{\alpha} \varphi_{w}\right\|_{-M}^{*} \leqslant C$ for such $M$, with $C$ independent of $w$ in $W$. Thus (3.7) implies the required estimate (3.6). This completes the proof of the second main result, Theorem $\mathrm{B}_{s}$.

4. Estimates for the $\bar{\partial}$-Neumann problem and for holomorphic functions. This section summarizes firstly some standard estimates for the $\bar{\partial}$-Neumann problem and secondly some special Sobolev estimates for holomorphic functions. A good reference for the $\bar{\partial}$-Neumann problem is [15]; details about Sobolev norms of holomorphic functions will appear in a forthcoming article [10].

The lemmas in this section should be understood to carry the following assumptions: $\Omega$ is a smooth bounded strictly pseudoconvex domain in $\mathbf{C}^{n}$; the functions $\zeta_{1}$ and $\zeta_{2}$ are real-valued cut-off functions in $C_{0}^{\infty}\left(\mathbf{C}^{n}\right)$ with $\zeta_{2}$ identically 1 in a neighborhood of the support of $\zeta_{1}$; and $M$ is an arbitrary positive integer. 
Consider $\bar{\partial}$ as a closed densely-defined operator from the Hilbert space of $(p, q)$-forms with square-integrable coefficients to the Hilbert space of $(p, q+1)$ forms with square-integrable coefficients. The Hilbert space adjoint of $\bar{\partial}$ is denoted $\bar{\partial} *$. The Neumann operator $N$ on $(0,1)$-forms is the inverse of $\bar{\partial} * \bar{\partial}+\bar{\partial} \bar{\partial} *$. It is well known that $N$ admits strong estimates in Sobolev norms (Kohn [17, 15]). Roughly speaking $N$ gains one derivative, the combination $\bar{\partial} * N$ gains one-half derivative, and the combination $\bar{\partial} * N \bar{\partial}$ preserves the number of derivatives. The formula $P_{0}=$ Id $\bar{\partial} * N \bar{\partial}$ for the Bergman projection, together with the estimates for $N$, leads to pseudolocal estimates for $P_{0}$.

LEMMA 4.1. For every nonnegative real number $r$ there is a constant $C$ such that

$$
\begin{aligned}
& \left\|\zeta_{1} P_{0} u\right\|_{r} \leqslant C\left(\left\|\zeta_{2} u\right\|_{r}+\|u\|_{-M}^{*}\right), \\
& \left\|\zeta_{1} P_{0} u\right\|_{-r}^{*} \leqslant C\left(\left\|\zeta_{2} u\right\|_{-r}^{*}+\|u\|_{-M}^{*}\right)
\end{aligned}
$$

for all $u$ in $C^{\infty}(\bar{\Omega})$. In particular

$$
\left\|P_{0} u\right\|_{r} \leqslant C\|u\|_{r}, \quad\left\|P_{0} u\right\|_{-r}^{*} \leqslant C\|u\|_{-r}^{*}
$$

The first inequality is well known. It is commonly written with ambient term $\|u\|_{0}$, but the stronger form given here follows from the usual proof. The second inequality, with negative indices, follows by duality.

An immediate corollary is the following local density statement for holomorphic functions.

LEMMA 4.2. Let $r$ and $s$ be positive real numbers such that $r \geqslant s$. If $h$ is a holomorphic function such that $h \in W^{s}(\Omega)$ and $\zeta_{2} h \in W^{r}(\Omega)$, then there is a sequence $h_{1}, h_{2}, \ldots$ of holomorphic functions in $C^{\infty}(\bar{\Omega})$ such that

(a) $\left\|h-h_{k}\right\|_{s} \rightarrow 0$, and

(b) $\left\|\zeta_{1}\left(h-h_{k}\right)\right\|_{r} \rightarrow 0$

as $k \rightarrow \infty$.

Proof. Let $\eta$ be a real-valued cut-off function in $C_{0}^{\infty}\left(\mathbf{C}^{n}\right)$ such that $\eta=1$ in a neighborhood of the support of $\zeta_{1}$ and $\zeta_{2}=1$ in a neighborhood of the support of $\eta$. Take a sequence $u_{1}, u_{2}, \ldots$ of functions in $C^{\infty}(\bar{\Omega})$ such that $u_{k} \rightarrow h$ in $W^{s}(\Omega)$ and $\eta u_{k} \rightarrow \eta h$ in $W^{r}(\Omega)$, and set $h_{k}$ equal to $P_{0} u_{k}$. It follows from Lemma 4.1 that the holomorphic functions $h_{k}$ have the required properties.

To state estimates for $\bar{\partial}^{*} N$ in norms with negative indices it is necessary to introduce tangential Sobolev norms. Each boundary point of $\Omega$ has a neighborhood in which it is possible to choose smooth real coordinates $t_{1}, \ldots, t_{2 n-1}, \rho$, where $\rho$ is a given defining function for $\Omega$. A function $u$ supported in such a boundary chart has tangential Fourier transform

$$
\hat{u}(\tau, \rho)=\int_{\mathbf{R}^{2 n-1}} e^{-i\langle t, \tau\rangle} u(t, \rho) d t
$$

and tangential Sobolev norm

$$
\|u\|_{r}^{2}=\int_{-\infty}^{0} \int_{\mathbf{R}^{2 n-1}}\left(1+|\tau|^{2}\right)^{r}|\hat{u}(\tau, \rho)|^{2} d \tau d \rho
$$


Of course norms of forms are defined componentwise. When $r>0$ the tangential norm is dominated by the usual Sobolev norm, but when $r<0$ this relation is reversed (by duality).

LEMMA 4.3. Let $u$ be a $(0,1)$-form with coefficients in $C^{\infty}(\bar{\Omega})$ such that $\bar{\partial} u=0$. If $\zeta_{1}$ and $\zeta_{2}$ are supported in a boundary chart then for every real number $r$

$$
\begin{aligned}
& \left\|\zeta_{1} \bar{\partial}^{*} N u\right\|_{r+1 / 2} \leqslant C\left(\left\|\zeta_{2} u\right\|_{r}+\|u\|_{-M}^{*}\right) \quad \text { if } r \geqslant 0, \\
& \left\|\zeta_{1} \bar{\partial}^{*} N u\right\|_{r} \leqslant C\left(\left\|\zeta_{2} u\right\|_{r-1 / 2}+\left\|\zeta_{2} \bar{\partial} * N u\right\|_{-M}+\|u\|_{-M}^{*}\right) \quad \text { if } r \leqslant 1,
\end{aligned}
$$

with $C$ independent of $u$.

The first case is well known. The second case comes not from duality but from the same techniques [15, p. 53] used to prove the first case. The proof is omitted.

The following lemmas concern Sobolev norms of holomorphic functions. The first one says that three different norms are equivalent.

LEMMA 4.4. If $h$ is a holomorphic function, then for every positive real number $r$

$$
\left\|\zeta_{1} h\right\|_{-r} \leqslant\left\|\zeta_{1} h\right\|_{-r}^{*} \leqslant C\left(\left\|\zeta_{2} h\right\|_{-r}+\|h\|_{-M}\right),
$$

and if $\zeta_{1}$ and $\zeta_{2}$ are supported in a boundary chart, then

$$
C^{-1}\left\|\zeta_{1} h\right\|_{-r} \leqslant\left\|\zeta_{1} h\right\|_{-r} \leqslant C\left(\left\|\zeta_{2} h\right\|_{-r}+\|h\|_{-M}\right)
$$

with $C$ independent of $h$.

To compute the Sobolev norm of a holomorphic function it turns out to be enough to consider derivatives in the direction normal to the boundary. Recall that if $\rho$ is a normalized defining function for $\Omega$, then $\partial / \partial \rho$ means $\Sigma\left(D_{j} \rho\right) D_{j}$.

LEMMA 4.5. If $h$ is a holomorphic function then for every real number $r$ and every positive integer $k$

$$
\left\|\zeta_{1} h\right\|_{r} \leqslant C\left(\left\|\zeta_{2}(\partial / \partial \rho)^{k} h\right\|_{r-k}+\|h\|_{-M}\right)
$$

with $C$ independent of $h$.

An inequality in the other direction also holds. In fact a differential operator of order $k$ maps $W^{r}(\Omega)$ continuously into $W^{r-k}(\Omega)$ except when $r=1 / 2,3 / 2, \ldots, k-$ $1 / 2$, and restricting to holomorphic functions eliminates the exceptions:

LEMMA 4.6. If $h$ is a holomorphic function and $L$ is a linear differential operator of order $k$ with coefficients in $C^{\infty}(\bar{\Omega})$, then for every real number $r$

$$
\left\|\zeta_{1} L h\right\|_{r} \leqslant C\left(\left\|\zeta_{2} h\right\|_{r+k}+\|h\|_{-M}\right)
$$

with $C$ independent of $h$.

The last three lemmas are actually true in a much more general setting. They hold for harmonic functions in arbitrary smooth bounded domains. The proofs use only standard elliptic theory (see [10] for details). 
5. Proof of the key local estimates. This section is devoted to the proof of Theorem 3.1 and the estimates (2.2) and (2.3). To keep track of the local behavior it is convenient to fix a sequence $\eta_{0}, \eta_{1}, \ldots$ of smooth real-valued cut-off functions such that $\eta_{0}=\zeta_{1}$, each $\eta_{j+1}$ is identically equal to one in a neighborhood of the support of $\eta_{j}$, and $\zeta_{2}$ is identically one on the support of $\eta_{j}$ for every $j$. It is also useful to introduce the notation

$$
\|\|_{q}^{(*)}=\left\{\begin{array}{lll}
\| & \|_{q} & \text { if } q \geqslant 0 \\
\| & \|_{q}^{*} & \text { if } q<0
\end{array}\right.
$$

in order to treat the cases of positive and negative indices together.

To prove (3.1) observe that

$$
\left\langle\zeta_{1} T_{s} u, \varphi\right\rangle_{0}=\left\langle u, P_{0}\left(\zeta_{1} \varphi\right)\right\rangle_{s}=\sum_{|\alpha| \leqslant s}\left\langle D^{\alpha} u, D^{\alpha} P_{0}\left(\zeta_{1} \varphi\right)\right\rangle_{0}
$$

for $u$ in $C^{\infty}(\bar{\Omega})$ and $\varphi$ in $C_{0}^{\infty}(\Omega)$. When $|\alpha| \leqslant s$ and $r \geqslant s$

$$
\begin{aligned}
\left|\left\langle D^{\alpha} u, D^{\alpha} P_{0}\left(\zeta_{1} \varphi\right)\right\rangle_{0}\right| \leqslant & \left\|\eta_{1} D^{\alpha} u\right\|_{r-s}\left\|D^{\alpha} P_{0}\left(\zeta_{1} \varphi\right)\right\|_{-(r-s)}^{*} \\
& +\left\|D^{\alpha} u\right\|_{0}\left\|\left(1-\eta_{1}\right) D^{\alpha} P_{0}\left(\zeta_{1} \varphi\right)\right\|_{0}
\end{aligned}
$$

By Lemmas 4.4 and 4.6 and the estimates of Lemma 4.1 for the Bergman projection, it follows that the first term on the right-hand side is at most a constant times

$$
\left(\left\|\zeta_{2} u\right\|_{r}+\|u\|_{-M}\right)\|\varphi\|_{-r+2 s}^{(*)}
$$

while by the disjointness of the supports of $\left(1-\eta_{1}\right)$ and $\zeta_{1}$ the second term is dominated by $\|u\|_{s}\|\varphi\|_{-M}^{*}$ for every positive $M$. Therefore

$$
\left|\left\langle\zeta_{1} T_{s} u, \varphi\right\rangle_{0}\right| \leqslant C\left(\left\|\zeta_{2} u\right\|_{r}+\|u\|_{s}\right)\|\varphi\|_{-r+2 s}^{(*)}
$$

Since $C_{0}^{\infty}(\Omega)$ is dense in both $W_{0}^{t}(\Omega)$ and $\left(W^{t}(\Omega)\right)^{*}$ when $t \geqslant 0$, it follows by taking the supremum over $\varphi$ such that $\|\varphi\|_{-r+2 s}^{(*)}=1$ that

$$
\left\|\zeta_{1} T_{s} u\right\|_{r-2 s} \leqslant C\left(\left\|\zeta_{2} u\right\|_{r}+\|u\|_{s}\right) \text {. }
$$

This proves the first inequality in Theorem 3.1 .

Inequality (3.2) for holomorphic functions follows from the same argument if in (5.1) the function $u$ is replaced by $h$ and the second term on the right-hand side is replaced by

$$
\left\|D^{\alpha} h\right\|_{-M-s}^{*}\left\|\left(1-\eta_{1}\right) D^{\alpha} P_{0}\left(\zeta_{1} \varphi\right)\right\|_{M+s} .
$$

Next consider inequality (3.3) for $h$ in $H^{s}(\Omega)$. If $\zeta_{1}$ has compact support in $\Omega$ then $\left\|\zeta_{1} h\right\|_{r} \leqslant C\|h\|_{-M}$, so after taking a partition of unity it may be assumed that $\zeta_{1}$ and $\zeta_{2}$ are supported in a boundary chart. Suppose for the moment it has been shown that $\eta_{j+4} h \in W^{q}(\Omega)$ for a certain index $j$ and a certain real number $q$ less than or equal to $r-1 / 2$. (This assumption holds for every $j$ when $q \leqslant s$.) In view of the local density statement of Lemma 4.2 there is a sequence $h_{1}, h_{2}, \ldots$ of holomorphic functions in $C^{\infty}(\bar{\Omega})$ such that $h_{k} \rightarrow h$ in $W^{s}(\Omega)$ and $\eta_{j+3} h_{k} \rightarrow \eta_{j+3} h$ in $W^{q}(\Omega)$ as $k \rightarrow \infty$. 
If $\varphi \in C_{0}^{\infty}(\Omega)$, then by Fubini's theorem

$$
\begin{aligned}
\left\langle\eta_{j+1} T_{s} h, \varphi\right\rangle_{0} & =\left\langle h, P_{0}\left(\eta_{j+1} \varphi\right)\right\rangle_{s}=\lim _{k \rightarrow \infty}\left\langle h_{k}, P_{0}\left(\eta_{j+1} \varphi\right)\right\rangle_{s} \\
& =\lim _{k \rightarrow \infty}\left\langle\eta_{j+1} T_{s} h_{k}, \varphi\right\rangle_{0} .
\end{aligned}
$$

Writing $T_{s}=P_{0} L^{2 s}$ and $P_{0}=\mathrm{Id}-\bar{\partial} * N \bar{\partial}$ gives

$$
\left\langle\eta_{j+1} T_{s} h_{k}, \varphi\right\rangle_{0}=\left\langle\eta_{j+1} L^{2 s} h_{k}, \varphi\right\rangle_{0}-\left\langle\eta_{j+1} \bar{\partial} * N \bar{\partial} L^{2 s} h_{k}, \varphi\right\rangle_{0} .
$$

The main point of the argument is that the second term on the right-hand side is lower order than the first term. Lemma 4.3 implies

$$
\begin{aligned}
\left|\left\langle\eta_{j+1} \overline{\mathrm{\partial}} * N \overline{\mathrm{\partial}} L^{2 s} h_{k}, \varphi\right\rangle_{0}\right| \leqslant & C\left(\left\|\eta_{j+2} \overline{\mathrm{\partial}} L^{2 s} h_{k}\right\|_{q-2 s}+\left\|\eta_{j+2} \overline{\mathrm{\partial}} L^{2 s} h_{k}\right\|_{q-2 s}\right. \\
& \left.+\left\|\zeta_{2} \bar{\partial} * N \bar{\partial} L^{2 s} h_{k}\right\|_{-M-2 s}+\left\|\bar{\partial} L^{2 s} h_{k}\right\|_{-M-2 s}^{*}\right)\|\varphi\|_{2 s-q-1 / 2}^{(*)}
\end{aligned}
$$

(One or the other of the first two terms in parentheses on the right-hand side is irrelevant, depending on whether $q-2 s$ is positive or negative.) Since $\bar{\partial} h_{k}=0$ and the commutator $\left[\bar{\partial}, L^{2 s}\right]$ is an operator of order $2 s$, it follows from Lemma 4.6 that the first term in parentheses is at most a constant times $\left\|\eta_{j+3} h_{k}\right\|_{q}+\left\|h_{k}\right\|_{-M}$. In view of the norm equivalence stated in Lemma 4.4, the second term in parentheses admits the same bound. The third term in parentheses is at most

$$
\left\|\zeta_{2} P_{0} L^{2 s} h_{k}\right\|_{-M-2 s}+\left\|\zeta_{2} L^{2 s} h_{k}\right\|_{-M-2 s} \leqslant C\left(\left\|T_{s} h_{k}\right\|_{-M-2 s}+\left\|h_{k}\right\|_{-M}\right) \text {, }
$$

and by estimate (3.2) already proved this is dominated by $\left\|h_{k}\right\|_{-M}$, as is the fourth term in parentheses. Thus

$$
\left|\left\langle\eta_{j+1} \bar{\partial} * N \bar{\partial} L^{2 s} h_{k}, \varphi\right\rangle_{0}\right| \leqslant C\left(\left\|\eta_{j+3} h_{k}\right\|_{q}+\left\|h_{k}\right\|_{-M}\right)\|\varphi\|_{2 s-q-1 / 2}^{(*)} .
$$

Since $\varphi$ has compact support

$$
\lim _{k \rightarrow \infty}\left\langle\eta_{j+1} L^{2 s} h_{k}, \varphi\right\rangle_{0}=\left\langle\eta_{j+1} L^{2 s} h, \varphi\right\rangle_{0} .
$$

In view of estimate (5.4) it follows by combining (5.2) with (5.3) that

$$
\left|\left\langle\eta_{j+1} L^{2 s} h, \varphi\right\rangle_{0}\right| \leqslant\left|\left\langle\eta_{j+1} T_{s} h, \varphi\right\rangle_{0}\right|+C\left(\left\|\eta_{j+3} h\right\|_{q}+\|h\|_{-M}\right)\|\varphi\|_{2 s-q-1 / 2}^{(*)} .
$$

Taking the supremum over compactly supported $\varphi$ such that $\|\varphi\|_{2 s-q-1 / 2}^{(*)}=1$ gives

$$
\left\|\eta_{j+1} L^{2 s} h\right\|_{q-2 s+1 / 2} \leqslant C\left(\left\|\eta_{j+1} T_{s} h\right\|_{q-2 s+1 / 2}+\left\|\eta_{j+3} h\right\|_{q}+\|h\|_{-M}\right) .
$$

Recall from Lemma 1.1 that the top order term of $L^{2 s}$ on holomorphic functions is $2^{s}(\partial / \partial \rho)^{2 s}$. In view of Lemma 4.5

$$
\begin{aligned}
\left\|\eta_{j} h\right\|_{q+1 / 2} & \leqslant C\left(\left\|\eta_{j+1}(\partial / \partial \rho)^{2 s} h\right\|_{q-2 s+1 / 2}+\|h\|_{-M}\right) \\
& \leqslant C\left(\left\|\eta_{j+1} L^{2 s} h\right\|_{q-2 s+1 / 2}+\left\|\eta_{j+2} h\right\|_{q-1 / 2}+\|h\|_{-M}\right) .
\end{aligned}
$$


Together with (5.5) this implies

$$
\left\|\eta_{j} h\right\|_{q+1 / 2} \leqslant C\left(\left\|\zeta_{2} T_{s} h\right\|_{r-2 s}+\left\|\eta_{j+3} h\right\|_{q}+\|h\|_{-M}\right) .
$$

If the right-hand side of (3.3) is finite, then (5.6) implies that $\eta_{j} h \in W^{q+1 / 2}(\Omega)$. By induction on $q$ (starting from the value $\min (s, r-1 / 2)$ ) it follows that $\eta_{j} h \in$ $W^{r}(\Omega)$ for every $j$; moreover (5.6) holds whenever $q \leqslant r-1 / 2$. The middle term on the right-hand side of (5.6) has the same form as the left-hand side, but with the index lowered by $1 / 2$. Iterating (5.6) at most $2[q+M+1]$ times lowers this index to $-M$, resulting in

$$
\left\|\eta_{j} h\right\|_{q+1 / 2} \leqslant C\left(\left\|\zeta_{2} T_{s} h\right\|_{r-2 s}+\|h\|_{-M}\right) .
$$

Now set $j$ equal to 0 and $q$ equal to $r-1 / 2$ to obtain the desired estimate (3.3).

Inequality (3.4) is an easy consequence of (3.3) and the relation $T_{s} U_{s}=P_{0}$ of Lemma 2.1. If $h \in H^{0}(\Omega)$, then by (3.3)

$$
\begin{aligned}
\left\|\zeta_{1} U_{s} h\right\|_{r} & \leqslant C\left(\left\|\zeta_{2} T_{s} U_{s} h\right\|_{r-2 s}+\left\|U_{s} h\right\|_{-M}\right) \\
& =C\left(\left\|\zeta_{2} h\right\|_{r-2 s}+\left\|U_{s} h\right\|_{-M}\right),
\end{aligned}
$$

so it remains only to estimate the global term in (5.7). Since $U_{s}$ is bounded from $H^{0}(\Omega)$ to $H^{s}(\Omega)$, inequality (5.7) implies in particular the global estimate

$$
\left\|U_{s} h\right\|_{M+2 s} \leqslant C\|h\|_{M}
$$

when $M \geqslant 0$. If $\varphi \in C_{0}^{\infty}(\Omega)$, then Fubini's theorem, equation (5.8), and the estimates for the Bergman projection yield

$$
\begin{aligned}
\left|\left\langle U_{s} h, \varphi\right\rangle_{0}\right| & =\left|\left\langle h, U_{s} \varphi\right\rangle_{0}\right|=\left|\left\langle h, U_{s} P_{0} \varphi\right\rangle_{0}\right| \\
& \leqslant C\|h\|_{-M-2 s}\left\|U_{s} P_{0} \varphi\right\|_{M+2 s} \leqslant C\|h\|_{-M-2 s}\|\varphi\|_{M} .
\end{aligned}
$$

Therefore

$$
\left\|U_{s} h\right\|_{-M} \leqslant C\|h\|_{-M-2 s}
$$

when $M \geqslant 0$, and so (5.7) implies (3.4).

This completes the proof of Theorem 3.1. The global a priori estimates (2.2) and (2.3) are an immediate corollary. First note that (3.2) and (3.4) imply that $T_{s}$ and $U_{s}$ map $H^{\infty}(\bar{\Omega})$ into itself. Setting $\zeta_{1}$ equal to 1 on $\Omega$ in (3.2) and (3.3) gives

$$
\left\|T_{s} h\right\|_{r-2 s} \leqslant C\|h\|_{r}, \quad\|h\|_{r} \leqslant C\left(\left\|T_{s} h\right\|_{r-2 s}+\|h\|_{-M}\right) .
$$

To get (2.2) it remains only to observe by (5.9) that for $h$ in $H^{\infty}(\bar{\Omega})$

$$
\|h\|_{-M}=\left\|U_{s} T_{s} h\right\|_{-M} \leqslant C\left\|T_{s} h\right\|_{-M-2 s} .
$$

Finally (2.3) follows from (2.2) by replacing $h$ with $U_{s} h$ and using that $T_{s} U_{s} h$ equals $h$ for $h$ in $H^{\infty}(\bar{\Omega})$.

6. Further remarks. (1) The full force of the strict pseudoconvexity of $\Omega$ is not used in the proofs. The key element is the knowledge that $\bar{\partial}^{*} N$ gains a fractional derivative, but the size of the gain is unimportant. Accordingly Theorems $\mathrm{A}_{s}$ and $\mathrm{B}_{s}$ hold more generally when $\Omega$ is a smooth bounded pseudoconvex domain such that 
its Neumann operator admits subelliptic estimates. David Catlin [11] has recently characterized such domains as being the domains of finite type, in the sense that the maximum order of contact of complex varieties with the boundary is finite. Examples of such domains are strictly pseudoconvex domains with $C^{\infty}$ boundary and weakly pseudoconvex domains with real analytic boundary. See [12] for further discussion of finite type conditions.

(2) In this paper I have considered the projection $P_{s}$ only for integral values of $s$, but it can equally well be defined for fractional $s$. This has some interest because $s$ equal to $1 / 2$ corresponds to the Szegö projection. Methods similar to the ones used here show that the Szegö projection admits regularity estimates in domains of finite type. This result will be proved in a forthcoming paper [10].

\section{BIBLIOGRAPHY}

1. R. A. Adams, Sobolev' spaces, Academic Press, New York, 1975.

2. N. Aronszajn, Theory of reproducing kernels, Trans. Amer. Math. Soc. 68 (1950), 337-404.

3. D. Barrett, Regularity of the Bergman projection on domains with transverse symmetries, Math. Ann. 258 (1982), 441-446.

4. S. R. Bell, Biholomorphic mappings and the $\bar{\partial}$-problem, Ann. of Math. 114 (1981), 103-113.

5. ___ A representation theorem in strictly pseudoconvex domains, Illinois J. Math. 26 (1982), $19-26$

6. S. R. Bell and H. P. Boas, Regularity of the Bergman projection and duality of holomorphic function spaces, Math. Ann. 267 (1984), 473-478.

7. S. R. Bell and D. Catlin, Boundary regularity of proper holomorphic mappings, Duke Math. J. 49 (1982), 385-396.

8. S. Bergman, The kernel function and conformal mapping, 2nd ed., Math. Surveys, no. 5, Amer. Math. Soc., Providence, R. I., 1970.

9. H. P. Boas, Holomorphic reproducing kernels in Reinhardt domains, Pacific J. Math. 112 (1984), 273-292.

10. Regularity of the Szegö projection in domains of finite type, in preparation.

11. D. Catlin, Boundary invariants of pseudoconvex domains, Ann. of Math. (in press).

12. J. P. D’Angelo, Real hypersurfaces, orders of contact, and applications, Ann. of Math. 115 (1982), $615-637$.

13. K. Diederich and J. E. Fornaess, Boundary regularity of proper holomorphic mappings, Invent. Math. 67 (1982), 363-384.

14. C. Fefferman, The Bergman kernel and biholomorphic mappings of pseudoconvex domains, Invent. Math. 26 (1974), 1-65.

15. G. B. Folland and J. J. Kohn, The Neumann problem for the Cauchy-Riemann complex, Ann. of Math. Studies, no. 75, Princeton Univ. Press, Princeton, N. J., 1972.

16. N. Kerzman, The Bergman kernel. Differentiability at the boundary, Math. Ann. 195 (1972), $149-158$.

17. J. J. Kohn, Harmonic integrals on strongly pseudoconvex manifolds. I, II, Ann. of Math. 78 (1963), $112-148 ; 79$ (1964), 450-472.

18. J. L. Lions and E. Magenes, Non-homogeneous houndary value problems and applications, Vol. I, Springer-Verlag, New York, 1972.

19. D. H. Phong and E. M. Stein, Estimates for the Bergman and Szegö projections on strongly pseudo-convex domains, Duke Math. J. 44 (1977), 695-704.

Departmint of Mathematics, Columbia University, New York, New York 10027

Current address: Department of Mathematics, Texas A \& M University, College Station, Texas 77843 